\title{
Pattern recognition with a digital holographic microscope working in partially coherent illumination
}

\author{
F. Dubois, C. Minetti, O. Monnom, C. Yourassowsky, J.-C. Legros, and P. Kischel
}

\begin{abstract}
We describe the implementation of the automatic spatial-frequency-selection filter for recognition of patterns obtained with a digital holographic microscope working with a partially coherent source. The microscope provides the complex-optical-amplitude field that allows a refocusing plane-by-plane of the sample under investigation by numerical computation of the optical propagation. By inserting a correlation filter in the propagation equation, the correlation between the filter and the propagated optical field is obtained. In this way, the pattern is located in the direction of the optical axis. Owing to the very weak noise level generated by the partially coherent source, the correlation process is shift invariant. Therefore the samples can be located in the three dimensions. To have a robust recognition process, a generalized version of the automatic spatial-frequency-selection filters has been implemented. The method is experimentally demonstrated in a two-class problem for the recognition of protein crystals. (C) 2002 Optical Society of America
\end{abstract}

OCIS codes: $\quad 090.0090,100.0100,180.0180,070.0070,090.1760,100.6740$.

\section{Introduction}

In digital holography the interference patterns are recorded with an electronic camera, and the holographic reconstruction is performed by numerical means. ${ }^{1}$ Several optical methods have been implemented to extract the phase and amplitude information about the object beam from the recorded interference pattern. ${ }^{2,3}$ With the phase and the amplitude information, the digital holography reconstruction simulates the optical-beam propagation by use of a discrete implementation of the Kirchhoff-Fresnel propagation equations. The digital holographic methods have been demonstrated in various experimental situations, ${ }^{4-12}$ and are very promising in optical microscopy, where the three-dimensional (3D) reconstruction capability overcomes the very limited depths of field due to the high magnifications. ${ }^{13-15}$ A very crucial point in digital holography is that the recorded information on the $3 \mathrm{D}$ scene is a two-dimensional complex-

F. Dubois (frdubois@ulb.ac.be), C. Minetti, O. Monnom, C. Yourassowsky, and J.-C. Legros are with the Université Libre de Bruxelles, Microgravity Research Center, 50 Avenue F. Roosevelt, CP165/62, B-1050 Brussels, Belgium. P. Kischel is with the Laboratoire de Biologie Moléculaire et Génie Génétique, Allée de la Chimie, 3, Campus du Sart-Tilman, Bât. B6, 4000 Liege 1, Belgium.

Received 9 November 2001; revised manuscript received 18 March 2002.

0003-6935/02/204108-12\$15.00/0

(C) 2002 Optical Society of America amplitude field that can be handled as a special case of two-dimensional images. In particular, patternrecognition methods by correlation can be implemented in view to detect objects that have $3 \mathrm{D}$ extension. This approach has been tested for the recognition of macroscopic objects having diffuse optical surfaces. ${ }^{16}$ In that case, the recorded holographic information is related to the specific speckle field diffused by the object and the shift-invariance property of the correlation is reduced. To overcome those limitations, the recognition by correlation on the irradiance of the digitally propagated holographic data and image-processing method to reduce the speckle noise were considered. ${ }^{17,18}$ In this case the sensitivity of the correlation with respect to specklefield phase is removed. However, it has been shown that the signal phase contains crucial information. ${ }^{19}$ Therefore the phase should be kept to achieve the full capabilities of pattern recognition in digital holography. In this contribution, we investigate the recognition by correlation of full complex-amplitude fields obtained by a digital holographic microscope. Holographic setups often use laser sources that avoid any coherence problems in interferometer configuration. However, highly coherent beams are very sensitive to the microstructure of the sample and to any defect in the optical paths. As a result, the complex amplitudes are deeply affected by the coherent noise that severely reduces the optical quality. ${ }^{20}$ To alleviate such problems, our microscope uses a partially coherent source. 
The microscope set up and the related optical methods are described in Section 2. To implement the recognition process by correlation for digital holographic signals, an impressive set of filtercomputation algorithms is available in the literature. Those methods overcome the classical limitations of the matched filters. ${ }^{21}$ With the synthetic discriminant function (SDF) algorithm, ${ }^{22}$ the filters are computed as linear combinations of reference patterns to give $a$ priori fixed central-correlation intensities or responses when they are correlated with the reference images. This algorithm has played a central role in making the recognition invariant to input distortions. Adding minimization criteria to the SDF central-correlation constraints made further significant improvements. The minimization of the mean square variance for input images corrupted by noise, ${ }^{23}$ and the minimization of the average correlation energy 24,25 (MACE) have been used to optimize, respectively, the signal-to-noise ratio (SNR) and the peak sharpness. Methods have been also successfully proposed to globally optimize several criteria. ${ }^{26-28}$ It has been also demonstrated that nonlinear transforms applied in the Fourier plane can significantly improve the discrimination, and the correlation noise robustness, ${ }^{29-31}$ and that this method can be applied when distortion invariance is requested. ${ }^{32}$ Critical reduction of the correlation height occurs when the targets are surrounded by noise or by clutter. This problem has been considered and elegant solutions based on the decision theory $^{33,34}$ or on a minimization of mean square error ${ }^{35}$ have been proposed. To make the recognition insensitive to the input distortions, we developed the automatic spatial frequency selection (ASFS) algorithm that involves reference images and distorted versions of them to select the significant spatial frequencies ${ }^{36-38}$ in the recognition process. The implementation of the correlation in the digital holographic process is described in Section 3. The ASFS method is described in Section 4, where the changes to be used with digital holographic amplitudes are outlined. With the ASFS algorithm, the central-correlation amplitudes between the reference images and the filter are constrained to give $a$ priori set values, and a cost function depending on the reference and distorted images is minimized. This function is called the ASFS cost function.

In Subsection 5.A, we describe the implementation of a two-class recognition process based on images extracted from an actual application in protein crystallization monitoring. Some information about the experimental system is given at the beginning of this section. In Subsection 5.B, the recognition results are provided, and it is shown that the system keeps the shift-invariant property and is able to recognize objects in depth. Concluding remarks are reported in Section 6.

\section{Digital Holographic Microscope Working with a Partially Coherent Illumination}

The optical setup is very similar to the one described in detail in Ref. 14, and we give here only a short

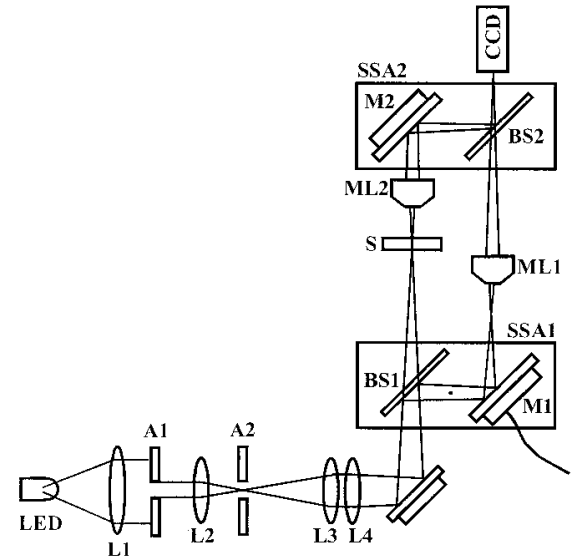

Fig. 1. Scheme of the microscope implemented in a MachZehnder interferometer with a spatially partial coherent source.

description. The microscope is implemented in a Mach-Zehnder interferometer (Fig. 1). A red lightemitting diode (LED) of $4 \mathrm{~mW}$ at $660 \mathrm{~nm}$ with a spectral width of $20 \mathrm{~nm}$ is used as an incoherent optical source. A secondary partially coherent source is achieved by imaging the LED-emitting surface with an optical filtering system. The collimated beam by the first lens $\left(\mathrm{L}_{1}\right.$-focal length $f_{1}$, is filtered by an aperture $\left(\mathrm{A}_{1}\right)$ of diameter $\rho$ that increases the spatial-coherence width of the source image in the back focal plane of the second lens $\left(\mathrm{L}_{2}\right.$ - focal length $f_{2}$ ). A third lens $\left(\mathrm{L}_{3}\right)$ collimates the beam and the lens $\left(\mathrm{L}_{4}\right)$ images the aperture $\mathrm{A}_{2}$ in the sample volume (S). The interferometer is constituted by two sub-system assemblies (SSA). Each SSA includes a beam splitter (BS) and a mirror (M). Microscope lenses $(10 \times)$ are placed in both the object and the reference beams. The image planes of these two microscope lenses (ML) are interfering on the input plane of the CCD camera sensor located after the beam combiner $\left(\mathrm{BS}_{2}\right)$. The partial coherence width of the secondary source is kept with a magnification ratio given by $f_{4} / f_{3}$, where $f_{4}$ and $f_{3}$ are the focal lengths of the lenses $\mathrm{L}_{4}$ and $\mathrm{L}_{3}$. The experimental volume is placed in the beam waist of the lens $\mathrm{L}_{4}$ in such a way that it is assumed that the sample $(\mathrm{S})$ is illuminated in plane waves. Indeed, the illumination wavefront of the sample can be considered as plane if the thickness of the sample is smaller than $2 \lambda f_{2}^{2} f_{4}{ }^{2} / \rho^{2} f_{3}{ }^{2}$ (see Ref. 14). In our system, $f_{2}=50$ $\mathrm{mm}, f_{3}=200 \mathrm{~mm}, f_{4}=200 \mathrm{~mm}$ and $\rho=0.25 \mathrm{~mm}$, the thickness has to be smaller than $52 \mathrm{~mm}$. We demonstrated that the partial coherence nature of the source limits the digital holographic refocusing distance $d$ according to the following inequality ${ }^{14}$ :

$$
d \ll \frac{2 f_{2} f_{4}}{\rho f_{3} v_{\max }},
$$

where $v_{\max }$ denotes the maximum expected spatial frequency.

For refocusing distance around the best-focus object plane higher than this limit, the resolution of the 
reconstructed image is progressively reduced. The main advantages in using a partially coherent source are the speckle removal, the influence reduction of perturbations that are located at a distance $d$ larger than the one expressed by the inequality of Eq. (1), and the suppression of multiple reflection interferences. We have a magnification ratio of 10 fixed by the microscope lenses. Thus $0.86 \mu \mathrm{m}$ of the input scene matches the CCD pixel size of $8.6 \mu \mathrm{m}$. Therefore $v_{\max }$ is estimated equal to $10^{6} / \mathrm{m}$ and $d$ has to be smaller than $400 \mu \mathrm{m}$. For refocusing distances higher than this limit, the resolution of the reconstructed image is progressively reduced. As the digitized images (256 levels) have $512 \times 512$ pixels, the field of view of the system is approximately $440 \mu \mathrm{m} \times$ $440 \mu \mathrm{m}$.

The mirror $\mathrm{M}_{1}$ is mounted on a piezoelectric transducer to implement the phase-stepping technique. The modulo $2 \pi$ phase $\phi(s, t)$, where $(s, t)$ are the discrete spatial variables, is computed from four $\pi / 2$ phase-shifted fringe images $I_{1}$ to $I_{4}$ with the fourframes algorithm:

$$
\phi(s, t)=\tan ^{-1}\left[\frac{I_{4}(s, t)-I_{2}(s, t)}{I_{1}(s, t)-I_{3}(s, t)}\right]
$$

The modulus of the amplitude of the optical field is calculated as the square root of the object intensity distribution.

\section{Digital Holographic Reconstruction and Correlation Implementation}

The object path of the optical system described in the previous section performs the image of the light distribution of the focused plane $\mathrm{P}$ that is crossing the experimental volume of the sample. As the interference patterns between the object and the reference beams are recorded with a phase-shifting capability, the complex amplitude in the focused plane is completely determined. Thanks to this information, we want to compute the optical field in planes that are parallel to $\mathrm{P}$, the focused plane, and that are also crossing the sample. The amplitude distribution in a plane $\mathrm{P}^{\prime}$ parallel to $\mathrm{P}$ and separated by a distance $d$ along the optical axis is computed by the KirchhoffFresnel propagation integral in the paraxial approximation, ${ }^{39}$

$$
\begin{aligned}
u_{0}\left(x^{\prime}, y^{\prime}\right)= & \exp (i k d)\left\{F _ { ( C ) v _ { x } , v _ { y } } { } ^ { - 1 } \operatorname { e x p } \left[-\frac{j k d \lambda^{2}}{2}\left(v_{x}^{2}\right.\right.\right. \\
& \left.\left.\left.+v_{y}{ }^{2}\right)\right]\left[F_{(C) x, y}{ }^{+1} u_{i}(x, y)\right]\right\}
\end{aligned}
$$

where $u_{i}(x, y)$ is a complex optical field in $\mathrm{P}, u_{0}\left(x^{\prime}, y^{\prime}\right)$ is the complex optical field in $\mathrm{P}^{\prime}, \lambda$ is the wavelength, $k=2 \pi / \lambda,(x, y),\left(x^{\prime}, y^{\prime}\right)$ are the spatial variables, $\left(v_{x}\right.$, $v_{y}$ ) are the spatial frequencies, $j=\sqrt{-1}$, and $F_{(C) \alpha \beta}{ }^{ \pm 1} g(\alpha, \beta)$ denotes the direct or inverse two- dimensional continuous Fourier transformations defined by

$$
\begin{aligned}
F_{(C) \alpha \beta}{ }^{ \pm 1} g(\alpha, \beta)= & \int_{-\infty}^{\infty} \int_{-\infty}^{\infty} \exp [\mp 2 j \pi(\alpha \eta \\
& +\beta \xi)] g(\alpha, \beta) d \alpha d \beta .
\end{aligned}
$$

For digital reconstruction, Eq. (3) is implemented in a discrete form. The sampling distance is $\Delta$ in both $x$ and $y$ directions. The discrete form of Eq. (3) is written by

$$
\begin{aligned}
u_{0}\left(s^{\prime} \Delta, t^{\prime} \Delta\right)= & \exp \{j k d\} F_{U, V}{ }^{-1} \exp \left[\frac { - j k \lambda ^ { 2 } d } { 2 N ^ { 2 } \Delta ^ { 2 } } \left(U^{2}\right.\right. \\
& \left.\left.+V^{2}\right)\right] F_{s, t}{ }^{+1} u_{i}(s \Delta, t \Delta),
\end{aligned}
$$

where $N$ is the number of pixels in both directions and $s, t, s^{\prime}, t^{\prime}, U$ and $V$ are integer numbers varying from 0 to $N-1$. $\quad F^{ \pm 1}$ denotes the direct and inverse Fourier transformation defined by

$$
\begin{aligned}
F_{k, l}{ }^{ \pm 1} g(m, n)= & \frac{1}{N} \sum_{k, l=0}^{N-1} \exp \left[\mp \frac{2 \pi j}{N}(m k\right. \\
& +n l)] g(k, l),
\end{aligned}
$$

where $k, l, m, n$ are integers in such a way that $k, l$, $m, n=0, \ldots, N-1$. The optical quality of the refocusing is shown on a microscopic scale by Figs. 2(a) and 2(b). The image of Fig. 2(a) is the intensity distribution recorded with a defocus distance of 400 $\mu \mathrm{m}$. Figure 2(b) shows the refocusing by digital holography. In the following, there is no need to consider amplitude distributions depending on continuous space and spatial frequencies variables $(x, y),\left(v_{x}, v_{y}\right)$. Therefore we simplify the notations by considering sampled amplitude distributions depending on integer spatial variables. In the spatial domain, the sampled amplitude distributions will be noted by the lower case letters $x(s, t), y(s, t)$, and $h(s$, $t$ ), where $s, t$ are integers $(s, t=0, \ldots, N-1)$ and $h$ represents a correlation filter as defined below. The corresponding amplitude distributions in the spatialfrequency domain are denoted by the corresponding uppercase letters $X(U, V), Y(U, V)$, and $H(U, V)$. Thanks to these notations, Eq. (5) can be rewritten as

$$
\begin{aligned}
x_{0}\left(s^{\prime}, t^{\prime}\right)= & \exp \{j k d\} F_{U, V}^{-1} \exp \left[\frac { - j k \lambda ^ { 2 } d } { 2 N ^ { 2 } \Delta ^ { 2 } } \left(U^{2}\right.\right. \\
& \left.\left.+V^{2}\right)\right] F_{s, t}{ }^{+1} x_{i}(s, t),
\end{aligned}
$$

where

$$
\begin{gathered}
x_{0}\left(s^{\prime}, t^{\prime}\right)=u_{0}\left(s^{\prime} \Delta, t^{\prime} \Delta\right), \\
x_{i}(s, t)=u_{i}(s \Delta, t \Delta) .
\end{gathered}
$$




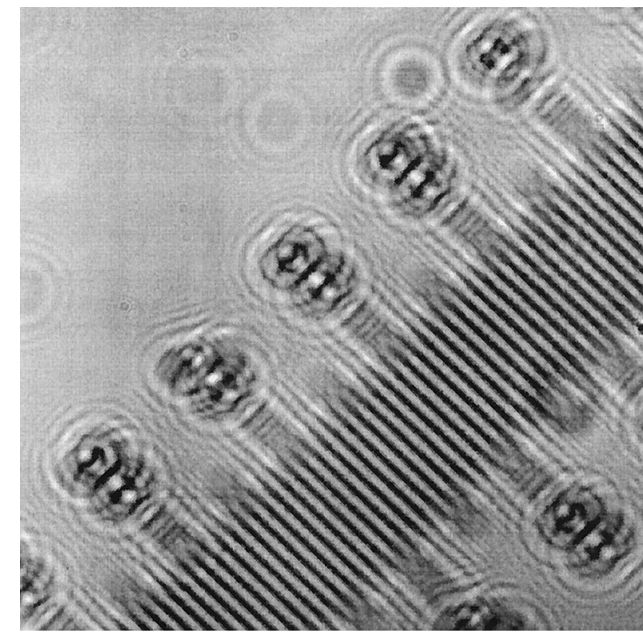

(a)

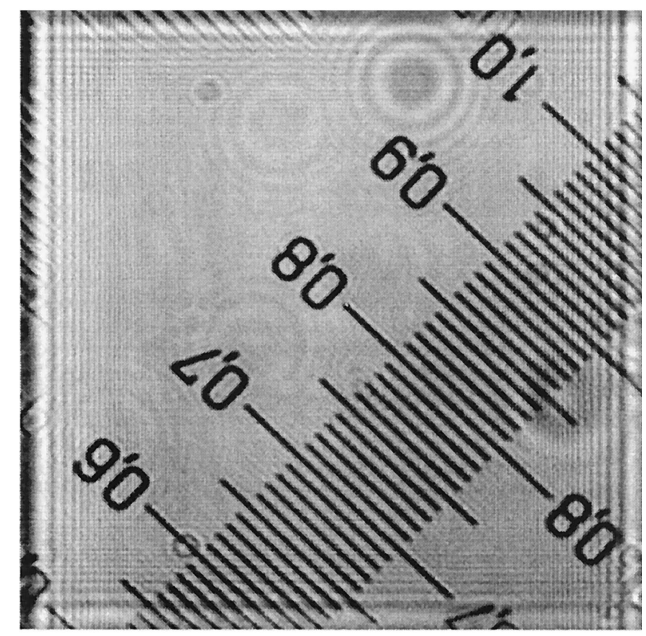

(b)

Fig. 2. Refocusing by digital holography on a metric scale (100 divisions $/ \mathrm{mm}$ ). (a) Image of the defocused intensity. (b) Image of the computer refocused intensity. The refocus distance is 400 $\mu \mathrm{m}$.

The correlation product $\left(x_{0} \otimes h\right)\left(s^{\prime}, t^{\prime}\right)$ between a filter $h(s, t)$ and an input amplitude distribution $x_{0}(s$, $t$ ) is achieved by computing:

$$
\left(x_{0} \otimes h\right)\left(s^{\prime}, t^{\prime}\right)=F_{U, V}{ }^{-1} H^{*}(U, V) X_{0}(U, V),
$$

where the superscript $*$ denotes the complex conjugate. By combining Eq. (7) and Eq. (9), the correlation product between propagated amplitude $x_{0}(s, t)$ by digital holography and a filter $h(s, t)$ is expressed by

$$
\begin{aligned}
\left(x_{0} \otimes h\right)\left(s^{\prime}, t^{\prime}\right)= & \exp \{j k d\} F_{U, V}{ }^{-1} H^{*}(U, V) \\
& \times \exp \left[\frac { - j k \lambda ^ { 2 } d } { 2 N ^ { 2 } \Delta ^ { 2 } } \left(U^{2}\right.\right. \\
& \left.\left.+V^{2}\right)\right] F_{s, t}{ }^{+1} x_{i}(s, t) .
\end{aligned}
$$

Equation (10) shows that the correlation product of the refocused amplitude plane with a correlation filter is achieved by introducing, as a multiplicative field, the complex conjugate of the filter amplitude in the digital holographic Eq. (7). The next section is devoted to the computational method of the correlation filters.

\section{Automatic Spatial Frequency Selection Algorithm to Compute Pattern Recognition Filters for Digital Holographic Patterns}

The ASFS algorithm that has been developed to compute correlation filter for image recognition ${ }^{33}$ can be applied for complex amplitude patterns obtained in digital holography with only a few changes. As for many other correlation algorithms, the ASFS filter is computed thanks to a set of typical patterns that have to be representative of the application. For example, if we want to recognize a specific object that can be rotated, the designer will select a set of images representing the object with different orientations. It is also necessary to add patterns that have to be unrecognized. In our example, those could be images of objects that we want to reject. On the basis of those images, a correlation filter is computed by the ASFS algorithm in such a way that images to be recognized will give rise to important intensity correlation peaks while images to be rejected will only give low-level intensities. In its simplest way, the recognition is based on the correlation-peak intensity. We consider a two-class problem where the patterns to be rejected are placed in a set of patterns called class 0 and all patterns to be recognized are placed in a second set called class 1 . We want to realize a unique filter $h(s, t)$ that is able to discriminate the patterns of class 1 with respect to the patterns of class 0 . The generalization to multiple filters is obvious. On the basis of the patternrecognition problem, reference images $x_{k q}(s, t)$ are selected where $k$ is the class number $(k=0,1)$ and $q$ is the image number in the class $k[q=0, \ldots, Q(k)-$ 1 , where $Q(k)$ is the number of pattern of the class $k]$. It is also assumed that the total number of reference images is $T$. Filter $h(s, t)$ has to give a set of responses $r_{k q}$ defined by the user that are the correlation amplitudes located at $(0,0)$ when the filter is correlated with $x_{k q}(s, t)$. In the Fourier space, it is expressed by

$$
r_{k q}=\sum_{U, V=0}^{n-1} H^{*}(U, V) X_{k q}(U, V) .
$$

As the number of pixels is larger than the number of reference images, Eq. (11) does not completely determine the filter components. Therefore an additional constraint can be imposed to reduce the sidelobe energies. For this purpose a set of distorted images $y_{k q i}(s, t)$ is associated with each reference image $x_{k q}$, where $i=0, \ldots, I_{k q}-1$, and $I_{k q}$ is the number of distorted patterns corresponding to $x_{k q}(s$, $t)$. The $y_{k q i}(s, t)$ images are chosen in the definition step of the pattern recognition problem. As an ex- 
ample, if the patterns to be recognized can be rotated, $y_{k q i}(s, t)$ images will be slightly rotated versions of $x_{k q}(s, t)$. The ASFS constraint minimizes, in average, the contributions of the spatial frequencies, where the components between $X_{k q}(U, V)$ and $Y_{k q i}(U$, $V)$ are significantly different. It is requested that the following expression be minimized:

$$
F=\sum_{U, V=0}^{N-1} \sum_{k, q, i}\left|H^{*}(U, V)\left\{X_{k q}(U, V)-Y_{k q i}(U, V)\right\}\right|^{2}
$$

The ASFS filter is computed by imposing the Eq. (11) and by minimizing Eq. (12). By lexicographically scanning $H(U, V), X_{k q}(U, V)$, and $Y_{k q i}(U, V)$ to make column vectors $\mathbf{H}, \mathbf{X}_{\mathrm{kq}}$ and $\mathbf{Y}_{\mathrm{kqi}}$, and by ordering vectors $\mathbf{X}_{\mathrm{kq}}$ side-by-side, we build the $X$ matrix. Eq. (11) is rewritten by the following matrix equation:

$$
X^{+} \mathbf{H}=\mathbf{r},
$$

where the superscript ${ }^{+}$denotes the Hermitian conjugate operation $\mathbf{r}$ is the column vector that involves the response elements $r_{\mathrm{kq}}$ with the same ordering sequence as for matrix $X$.

The ASFS cost function of Eq. (12) is rewritten in a matrix form:

$$
F=\mathbf{H}^{+} E \mathbf{H} .
$$

$E$ is an $N^{2} \times N^{2}$ diagonal matrix defined by

$$
E(Z, Z)=\sum_{k, q, i}\left|X_{k q}(Z)-Y_{k q i}(Z)\right|^{2},
$$

where $X_{k q}(Z)$ and $Y_{k q i}(Z)$ are the $Z$ th components of vectors $\mathbf{X}_{\mathrm{kq}}$ and $\mathbf{Y}_{\mathrm{kqi}}$. Vector $\mathbf{H}$, which verifies Eq. (13) and which minimizes Eq. (14), is obtained by use of the method of the Lagrange multipliers ${ }^{24}$ and is expressed by

$$
\mathbf{H}=E^{-1} X\left(X^{+} E^{-1} X\right)^{-1} \mathbf{r} .
$$

Equation (16) is the solution of the ASFS filter.

The difference between the ASFS filters computed for digital holography and the ones for image processing is the full complex nature of the patterns $x_{k q}(s, t)$ and $y_{k q i}(s, t)$. For this reason, in the following $x_{k q}(s$, $t)$ and $y_{k q i}(s, t)$ will be called reference fields and distorted fields instead of reference and distorted images. It is also for this reason that the matrix $\left(X^{+} E^{-1} X\right)^{-1}$ is a complex matrix instead of a real symmetric one.

\section{Implementation of a Pattern-Recognition Problem}

\section{A. Statement of the Problem}

The pattern-recognition problem that we selected comes from an actual application in protein crystal growth. The reason why the protein crystal growth is a crucial issue is summarized as follows: The biochemical properties of a protein are mainly defined by its reactive sites and its atomic structure. The reactive-sites and the atomic-structure information are obtained by x-ray diffraction analysis of the pro- tein crystals. Owing to the structural complexity of proteins, the physical-chemical processes governing the crystallization of protein are badly or not at all known, obliging biochemists to use empirical methods. That means that they have to perform many crystallization processes with small changes of the experimental parameters before achieving success. As the crystal quality is first assessed by long opticalmicroscopy observations, there is an important need in automatic methods to monitor protein-crystalgrowth processes. In this way, the digital holographic microscope gives access to the localization of crystals in solution while the pattern-recognition techniques are intended to identify and track each crystal during growth. In our experiments, we use a lysozyme protein for the crystallization process. Our crystallization cells have an experimental volume surrounded by two parallel optical plates separated by an internal distance of $800 \mu \mathrm{m}$. Crystallization of the lysozyme protein solution, used at a concentration of $100 \mathrm{mg} / \mathrm{ml}^{-1}$, was initiated with a precipitating solution composed of sodium chloride $(20 \% \mathrm{w} / \mathrm{v}, \mathrm{pH} 4.5)$. Both precipitating and protein solutions were cast with $1 \%(\mathrm{w} / \mathrm{v})$ low melting agarose gel (SeaPlaque Agarose, Cambrex) above the gelling point $37^{\circ} \mathrm{C}$. The lysozyme gel was poured over the gelled precipitating solution. Owing to the distance between the optical windows, the growing crystals can be largely out of focus. In the example of the pattern-recognition problem that we tested, we want to reject the crystal shown by Figs. 3(a) and 3(b) (Crystal 0) and to recognize the crystal (Crystal 1) shown by the Figs. 4(a) and 4(b). Those pictures have been recorded when the crystals are focused by the imaging channel of the microscope. The phase images Figs. 3(b) and 4(b) are digitized on 256 levels. The actual phase value in one point is obtained by multiplying the corresponding gray level by the constant $2 \pi / 255$. In the phase images shown by Figs. $3(\mathrm{~b})$ and 4(b), we observe that the background phases around the crystals are not uniformly distributed owing to two types of optical defects. The optical windows of the experimental cell are not completely flat and are not exactly parallel to each other. There is also a small misalignment of the interferometer. Those optical defects have to be taken into account as they are introducing distortions and shifts on the Fourier transformations of the complex amplitudes during the correlation process. Therefore if no care is taken for that, we could expect significant misalignment between the Fourier-transformed input amplitude and the pattern-recognition filter. This problem was identified a long time ago as a critical one in optical implementation of the correlation. ${ }^{40}$ We could think that those defects could be removed by using higher-quality experimental cells and by adjusting the interferometer in a better way. However, this approach is not realistic from a practical point of view because large-scale experiment batches request cheap experimental cells that present significant deviations with respect to perfect flatness and thickness. We apply a phase correction on all the 


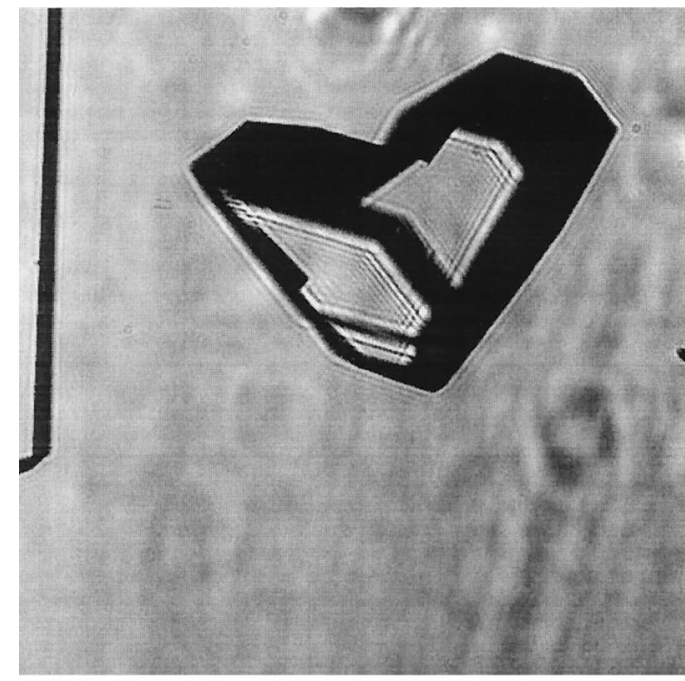

(a)

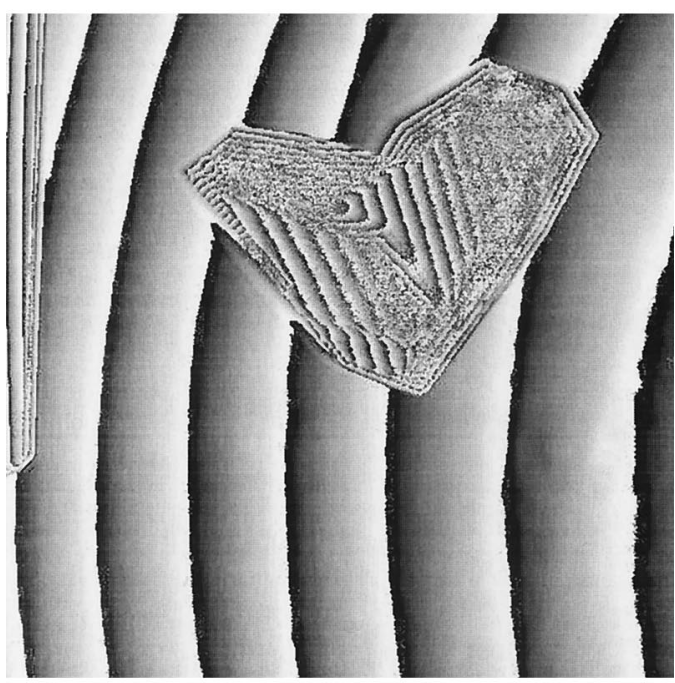

(b)

Fig. 3. (a) Intensity image and (b) phase image of the crystal to be rejected.

complex-amplitude fields that we are recording before any further processing. This is applied to the amplitude fields used to compute the correlation filter and for any amplitude fields recorded during the recognition process. Let us call $\phi(s, t)$ the phase of the complex amplitude $x(s, t)$, and $\phi_{B}(s, t)$ the phase of the background complex amplitude $x_{B}(s, t)$ measured with the cell without a sample. The corrected phase $\phi_{c}(s, t)$ is obtained by computing:

$$
\phi_{c}(s, t)=\bmod _{2 \pi}\left[\phi(s, t)-\phi_{B}(s, t)\right] .
$$

The corrected phase map corresponding to Fig. 4(b) is shown by Fig. 5 . In the application that we foresee, the phase-map correction is not really a problem. Indeed, before we begin an experimental crystal growth process, the phase map of the cell can be recorded as a background phase map used to correct the complex-amplitude fields during the process.

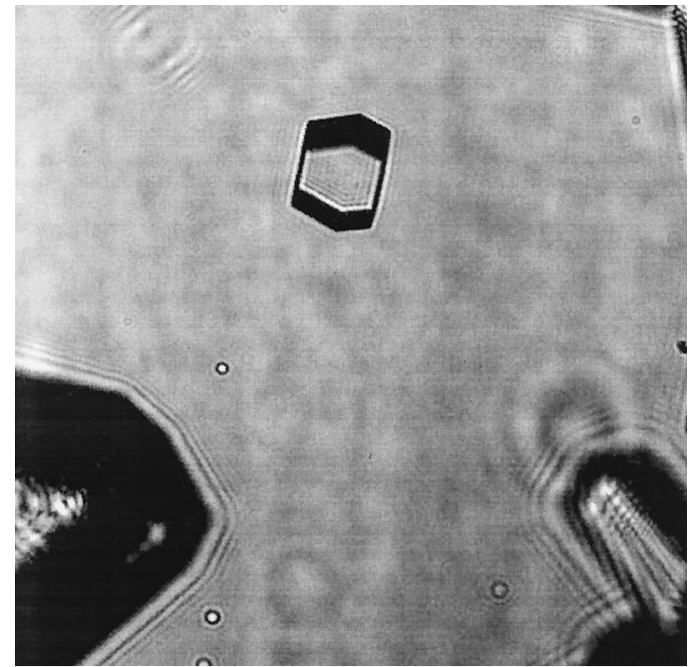

(a)

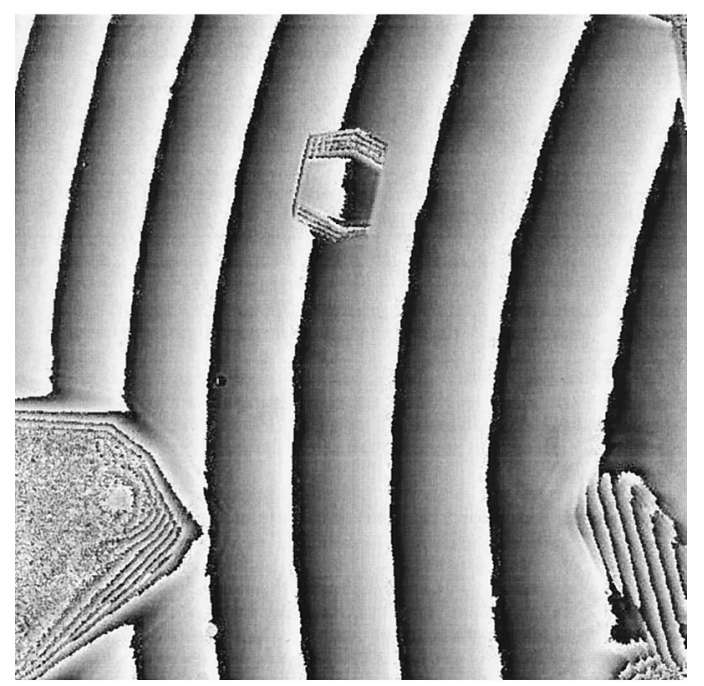

(b)

Fig. 4. (a) Intensity image and (b) phase image of the crystal to be recognized.

That can be also made with a set of background phase maps if the cell has to be laterally moved to monitor a larger area of the experimental cell. In our tests, we observed that the phase distortions in the cell itself due to the growing process could be neglected in such a way that a phase-correction map can be used during a complete growing process. Let us now describe how the reference-field sets have been constituted. For the first time, we formed two complexamplitude fields that we called primary-reference fields. They are formed according to the following operations. The intensity and corrected-phase images of the crystals in Figs. 3 and 4 have been cropped and centered in constant intensity and a phase background of $512 \times 512$ pixels. The constant intensity and the phase-background levels have been computed by averaging the intensities and corrected phases in a $20 \times 20$ window located in the background regions. The results of these processes for 


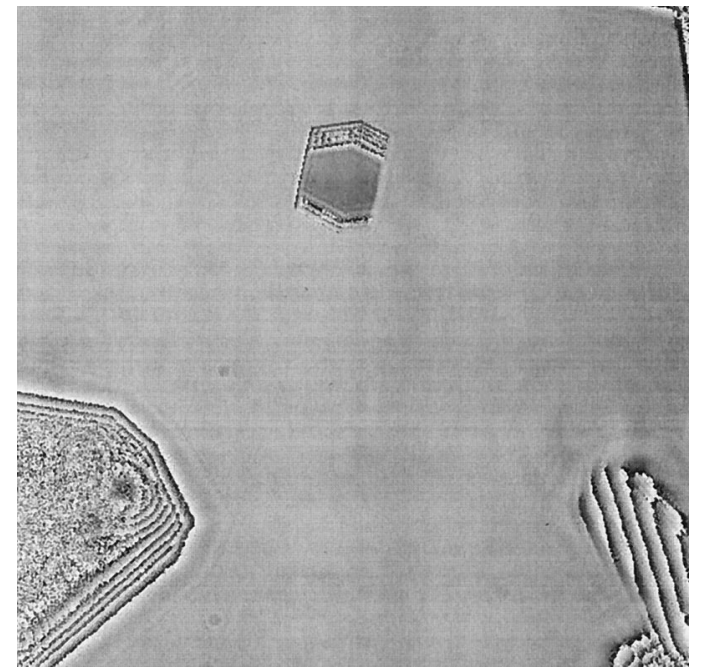

Fig. 5. Phase-corrected image of the crystal of Fig. 4(b).

the crystal of Fig. 4 are shown by the Figs. 6(a) and 6(b). We denote the intensity fields corresponding to the images in Figs. 3(a) and 4(a) by $I_{0}(s, t)$ and $I_{1}(s, t)$ and the corresponding corrected-phase fields by $\phi_{0}(s$, $t)$ and $\phi_{1}(s, t)$. The primary-reference fields are obtained by computing:

$$
x_{k}(s, t)=\sqrt{I_{k}(s, t)} \exp \left\{j \phi_{k}(s, t)\right\},
$$

where $k=0,1$.

From each $x_{k}(s, t)$, we build six reference fields $x_{k q}(s, t)$ by in-plane rotating $x_{k}(s, t)$ around the center point $(s, t)=(255,255)$ according to:

$$
x_{k q}(s, t)=R\left[-10^{\circ}+q .4^{\circ}\right] x_{k}(s, t),
$$

where the integer $q=0, \ldots, 5$ and $R[\alpha]$ denotes the rotation operation by an angle $\alpha$. To each reference pattern $x_{k q}(s, t)$ is associated with a single distorted pattern $y_{k q i}(s, t)$ in such a way that:

$$
y_{k q i}(s, t)=R\left[2^{\circ}\right] x_{k q}(s, t)
$$

because we have a single distorted field by reference field. In this case $i=0$.

The reasons why the selected distortions to compute the filter are from the in-plane rotation can be summarized as follows: The recognition of the pro-

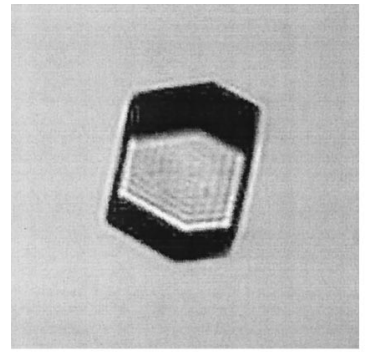

(a)

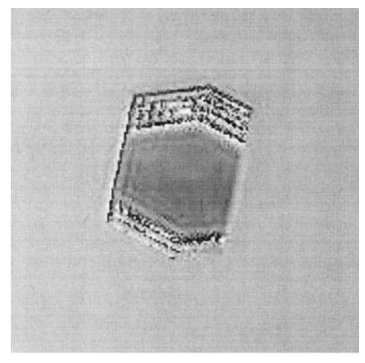

(b)
Fig. 6. Central region of the primary reference (a) intensity image and (b) phase image of the crystal to be recognized. tein crystal is a milestone of a wider objective that is the monitoring of growing crystals. For this purpose, pattern recognition will be used as a tracking tool that will allow us to identify and to follow several crystals in time. For each crystal, this objective requires the implementation of an iterative sequence of several processes including pattern recognition, postsegmentation to crop the crystal fields, and computation of a new pattern-recognition filter to be ready for a new identification later. Therefore the shape of each crystal is expected to change between two recordings in such a way that pattern recognition has to be robust with shape changes. The changes that can be expected are shifts, in-plane/out-of-plane rotations (when the crystals are growing in a solution instead of a gel), and growing. Growing can be anisotropic and very complex when crystal defects appear. Therefore prediction of these shape changes is not obvious and the most realistic way to handle this problem is to select an image-acquisition rate that guarantees small shape changes between two consecutive image acquisitions. In that context, small in-plane rotation as a distortion model increases the robustness of the recognition process due to the spatial-frequency selection obtained by the minimization of Eq. (12). The number of reference and distorted fields and the incremental angles between the reference fields can of course be optimized in view of a practical application. However, the scope of this article is not to address this problem: that will be the topic for a future work. Because of the fields $x_{k q}(s, t)$ and $y_{k q}(s, t)$, the filter $H(U, V)$ is computed according to Eq. (16). The filter components $H(U, V)$ are scaled in such a way that the response- 1 correlation peaks give rise to correlationpeak intensities equal to 255 in the center of the correlation plane when they are correlated with the reference fields to be recognized.

\section{B. Pattern Recognition Tests}

The first objective of the tests is to demonstrate that the pattern-recognition system, combining correlation and digital holographic reconstructions, is able to recognize the crystal of Figs. 4(a) and 4(b) and to reject the crystal of Figs. 3(a) and 3(b) even when the input fields are recorded out of focus in the microscope channel. If there is some defocus distance $D$ between the reference fields used to compute the filter and the input field, it is expected that a recognition correlation peak is happening when the digital holographic reconstruction distance of Eq. (10) is $d=$ $D$. Conversely, by scanning the reconstruction distance $d$ in Eq. (10), it is expected that we will observe a peak formation in depth with a maximum when the reconstruction distance $d$ corresponds to the defocus distance $D$. The second objective is to test the shiftinvariance property of the recognition process. Indeed, with respect to the pattern-recognition methods developed for image processing, we must work with important phase information directly recorded with the input images and we must check that the exper- 


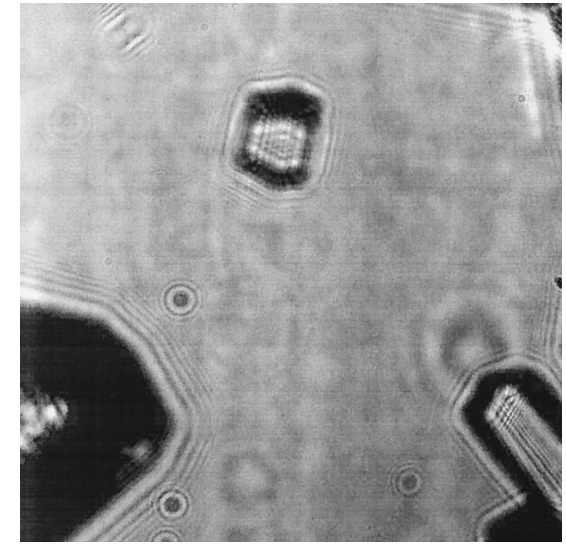

Fig. 7. Defocused image by a distance of $-150 \mu \mathrm{m}$.

imental process does not disturb it too much for the pattern-recognition operation.

To test these two aspects, we recorded intensity images and phase maps of the two crystals in two different lateral positions (displacement perpendicular to the optical axis) and for 10 defocus distances by changing, with mechanical translation stages, the position of the experimental cell in the sample place of

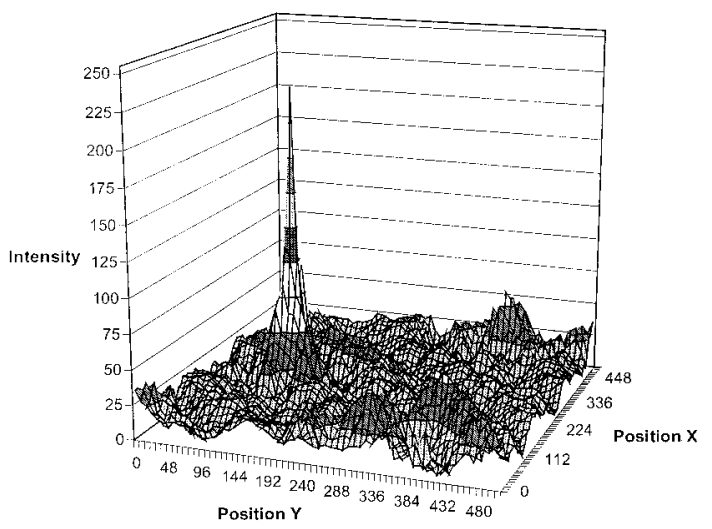

(a)

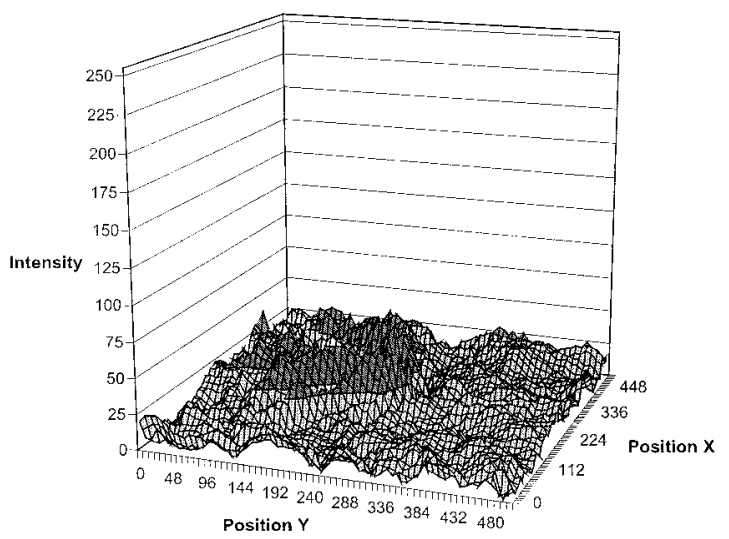

(b)

Fig. 8. Correlation intensities for the crystal to be recognized (a) series 3 and (b) series 1. Defocus distance $100 \mu \mathrm{m}$, reconstruction distance $100 \mu \mathrm{m}$.

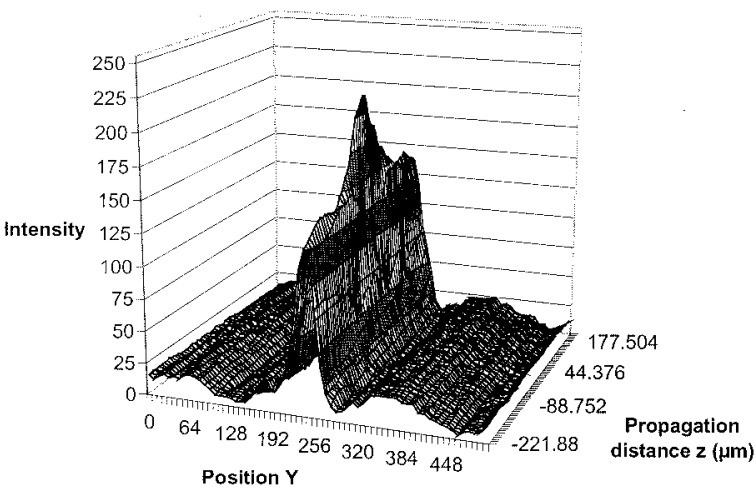

(a)

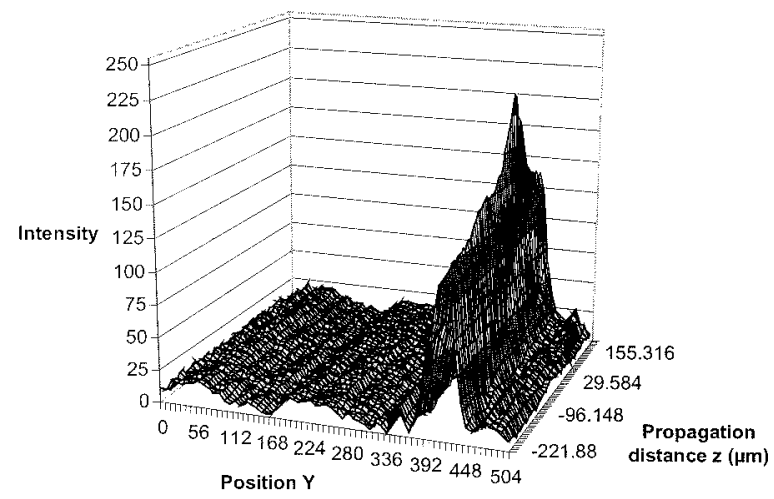

(b)

Fig. 9. Horizontal correlation-intensity profile crossing the correlation peak as a function of the digital holographic reconstruction distance $d$ (a) series 3 and (b) series 4 . Defocus distance $D=0$ $\mu \mathrm{m}$.

the microscope. Those images are sorted in four series according to the crystal number and the lateral position in the field of view. The series are labeled as follow: series 1 (crystal 0/lateral position 1), series 2 (crystal $0 /$ lateral position 2 ), series 3 (crystal 1/lateral position 1) and series 4 (crystal 1/lateral position 2). Note that each series involves different defocus distances $D$ of the same sample in the same position. The intensity and phase images were recorded without any processing of the background to operate in actual experimental conditions. The positions of the crystals with respect to the frame center are for the series $1,2,3$, and 4 , respectively, $(50,85)$, $(-49,-93),(-8,-122)$, and $(167,-115)$. The defocus distances that are considered for the four series cover a range of $225 \mu \mathrm{m}$ and start from $-150 \mu \mathrm{m}$ up to $75 \mu \mathrm{m}$ in steps of $25 \mu \mathrm{m}$. It has to be emphasized that a classical optical microscope with such magnification $(\times 10)$ has a typical depth of focus of $10 \mu \mathrm{m}$. A defocused intensity image (the crystal of Fig. 4) by a distance of $150 \mu \mathrm{m}$ is shown in Fig. 7 and it can be compared with the best focused image shown by Fig. 4(a). A correction-phase step has been applied with a single background phase for each series to keep a realistic experimental protocol. With the intensity images and the corrected phase images, the complexamplitude fields were computed for all the images of 


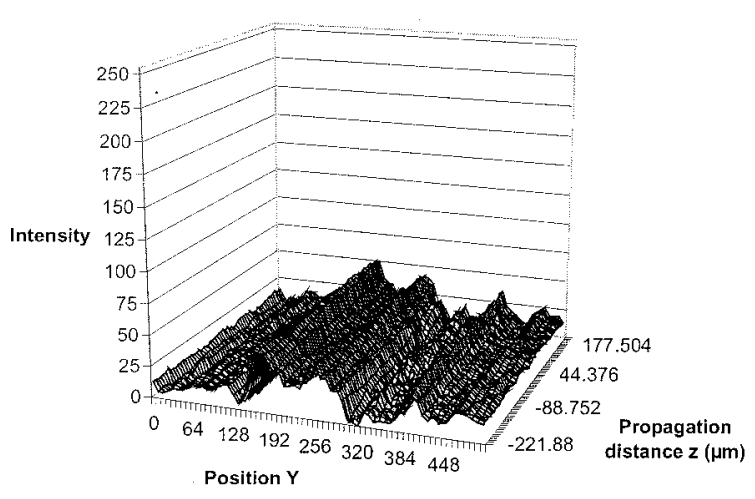

(a)

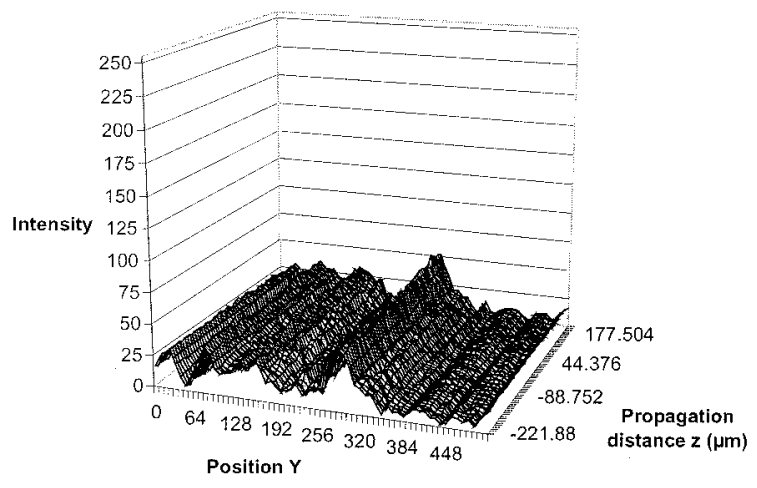

(b)

Fig. 10. Horizontal correlation intensity profile crossing the correlation center as a function of the digital holographic reconstruction distance $d$ (a) series 1 and (b) series 2. Defocus distance $D=$ $0 \mu \mathrm{m}$.

the four series. Correlation tests have been performed on the complete set of fields of the four series. The 3D plots of Figs. 8(a) and 8(b) show the correlation intensities when the filter is respectively correlated with the fields of series 3 and series 1 , when the defocus distance $D$ is in both case equal to $100 \mu \mathrm{m}$, and the reconstruction distance $d=100 \mu \mathrm{m}$. We observe in those plots that the correlation gives rise to quite-higher intensity levels for the crystal that has to be recognized. The recognition peak is also narrow and well shaped. We observe also that the maximum correlation peak is lower than the level of 255 constrained during the filter computation. This is due to the fact that the input field originates now from a real experimental process and not a reference field used to compute the filter. The 3D plots of Figs. 9 and 10 show, for the four series, the evolution of the horizontal correlation-intensity profile crossing the center of the crystal position (defined by the position of the reference fields) for various reconstruction distances $d$ of Eq. (10). In the recognition cases, we observe that the correlation-intensity profiles have a well-shaped maxima for $d$ values very similar to $D$ and that the intensity peak is sensitive with respect to the reconstruction distance $d$. Plots of Figs. 11 and 12 represent, for all the input fields of the four series, the evolution of the maximum correlation intensities as a function of the digital holographic reconstruction $d$. As expected, we observe that we obtain the maximum correlation intensities at reconstruction distances that correspond, within the accuracies of our mechanical translation stage, to the defocus distances of the recorded input images. We observe, also on these plots, similar results for the series 3 and 4 indicating that the system presents a good shift invariance property. Figure 13 shows the defocus distance $D$ as a function of the reconstruction distance $d$ when the correlation intensity has a maximum (series 3). Within the accuracy of our mechanical translation stage, we obtain a good linearity between $d$ and $D$, and a very small systematic error probably due to our mechanical translation stage. For the higher defocus distances $D$ in series 3 and 4, a low decrease of the correlation-peak height can be seen. The intensities corresponding to series 1 and 2 are quite lower and are almost identical for every

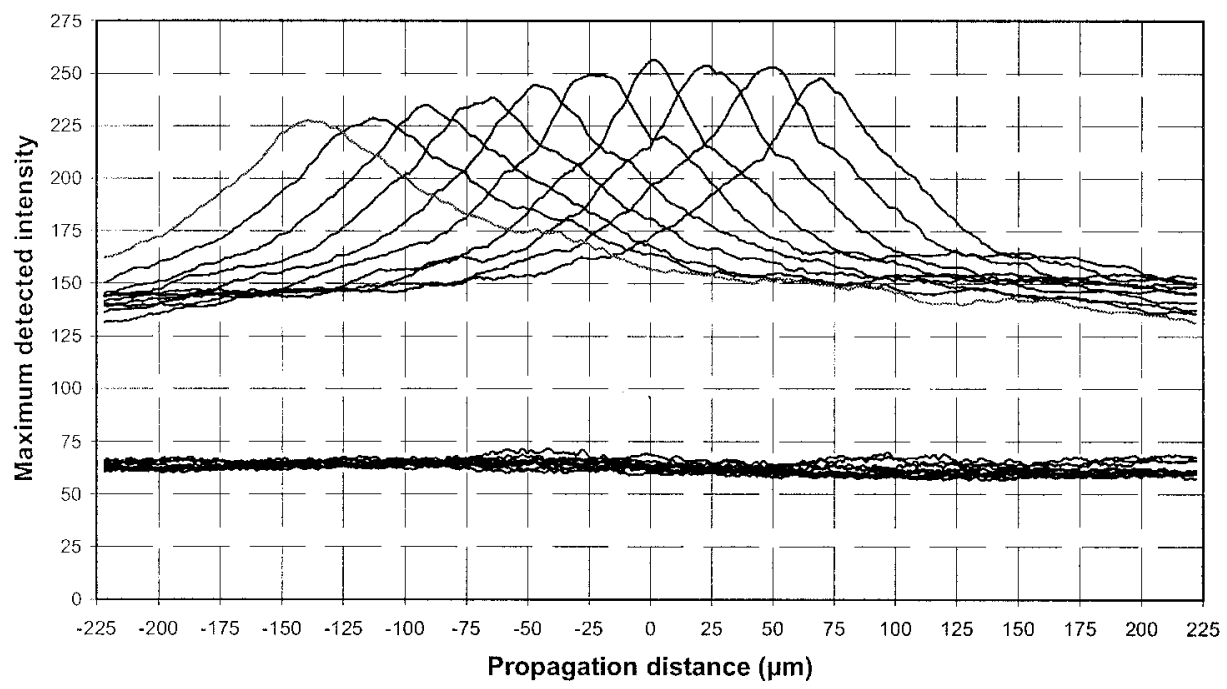

Fig. 11. Maximum correlation intensities as a function of the digital reconstruction distance $d$ for the samples of series 1 and 3 . 


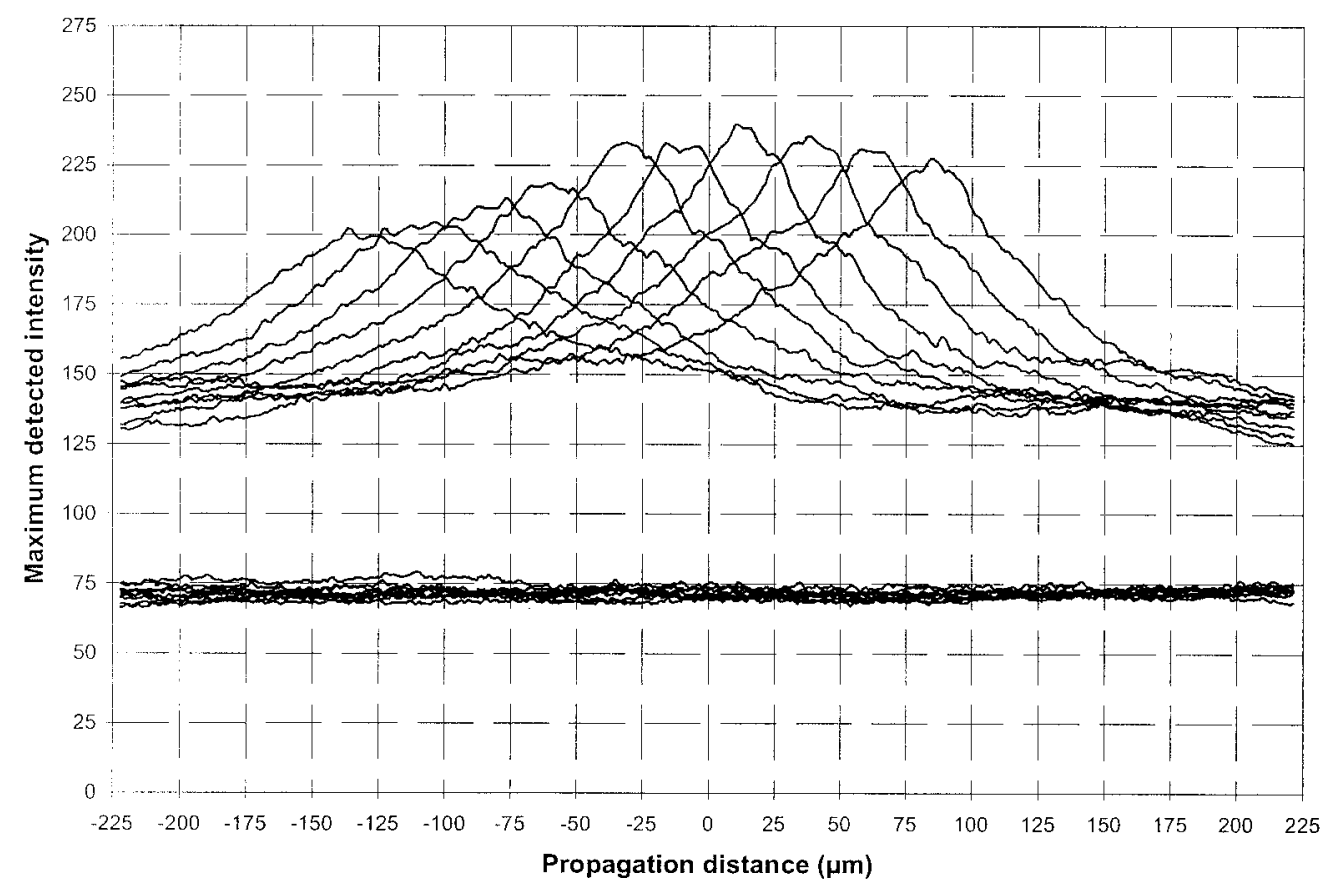

Fig. 12. Maximum correlation intensities as a function of the digital reconstruction distance $d$ for the samples of series 2 and 4 .

distance $d$. Therefore, we can apply a decisionthreshold level that correctly discriminate the results obtained with series 1 and 2 and series 3 and 4 . It can be objected that the recognition problem that we consider is obvious as the crystal shapes shown by Figs. 3 and 4 are very different. Indeed, a tracking application would include $a$ priori during any filter computation process a crystal to be recognized and a set of crystals to be rejected. To estimate the behavior of the pattern-recognition process with nontraining crystal samples, we measured the correlation-peak intensities for a set of 25 different fields that were not involved in the training set for the filter computation. As result, we have an average maximum correlation intensity of 64.30 with a standard deviation of 13.40 , indicating a good discrimination of the process for the recognition of the

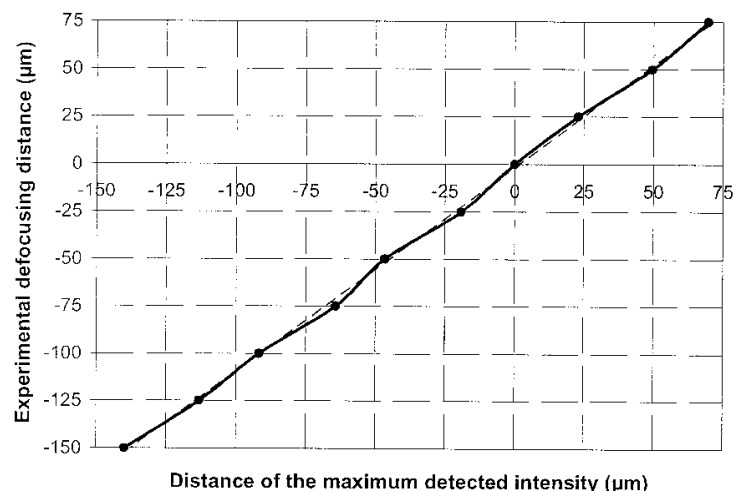

Fig. 13. Defocus distance $D$ as a function of the reconstruction distance $d$ when the maximum correlation intensity is reached for the samples of series 3 . crystal of Fig. 4. According to Eq. (19), the ASFS filter is computed to be robust with respect to in-plane rotations over a range of $\left[-10^{\circ}, 10^{\circ}\right]$ with respect to the crystal orientations shown by Figs. 3 and 4 . We tested this robustness by computing the intensities of the correlations between the filter and the in-plane rotated amplitude fields of the focus phase-corrected amplitudes corresponding to the crystals shown by Figs. 3 and 4 . The results are shown by Fig. 14 where the maximum correlation intensities are represented as a function of the in-plane rotation angle by a step of $1^{\circ}$ in the range $\left[-15^{\circ}, 15^{\circ}\right]$. We obtain two curves. The highest one corresponds to the amplitude fields to be recognized and the lowest one is obtained with the fields to be rejected. We observe that the intensity levels are quite different for the two

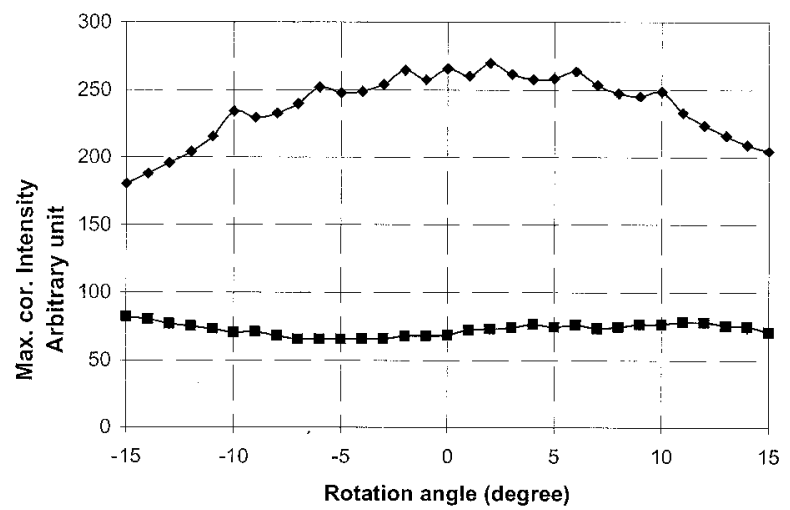

Fig. 14. Maximum correlation intensities between the ASFS filter and the in-plane rotated amplitude fields of the focus phasecorrected amplitudes corresponding to the crystals shown in Figs. 3 and 4. 
types of fields and that the fields to be recognized give rise to high-intensity levels for the orientations in the range of $\left[-15^{\circ}, 15^{\circ}\right]$ demonstrating that the ASFS filter responses are in agreement with the constraints imposed during the filter computation.

\section{Concluding Remarks}

This contribution describes a way to introduce pattern recognition by a correlation process on complete amplitude distributions produced by a digital holography microscope. In order to reduce the noise inherent to the laser source, we are using a digital holographic microscope that takes advantage of a partially coherent source. The ASFS algorithm has been implemented to compute the correlation filter. Owing to the low level of noise produced by the partially coherent source, the full amplitude of information of the input field is kept for the recognition process. However, the need of phase correction on every input pattern is identified. The pattern recognition by correlation on amplitude fields provided by the digital holographic setup is tested on a two-class problem for the recognition of protein crystals. It is shown that the system keeps the shift-invariance property of the usual correlation and that the maximum correlation peak occurs when the defocus distance of the object is equal, within the experimental errors, to the digital holographic reconstruction distance. Therefore the system is able to locate objects in depth when they are out of focus with respect to the imaging channel of the microscope. Future studies will be oriented toward the development of the automatic tracking of growing crystals for the implementation of an automated application. The implementation of the automated tracking system will be carefully studied as we can expect, owing to the growing process, to measure large phase changes between two consecutive hologram recordings of the same crystal. The basic concept of the tracking system is to compute, for each crystal, with a time delay $\Delta t$, a correlation filter based on a few previously recorded digital holograms for recognition. If the time delay is too long, the amplitude field of a crystal will be changed too much with respect to the previously recorded amplitude fields to enable a reliable recognition. Therefore the tracking will be implemented by adequately choosing the acquisition frequency of the digital holograms.

This research is supported by the Walloon Region Research Program "Recherche d'initiative" under the MICADO contract 001/4512. The authors are grateful to J. Becker from the European Space Agency who provided us the results of the pioneer works on digital holography obtained by B. Skarman.

\section{References}

1. U. Schnars and W. Jüptner, "Direct recording of holograms by a CCD target and numerical reconstruction," Appl. Opt. 33, 179-181 (1994).

2. K. Creath, "Temporal Phase Measurement Methods," in Interferogram Analysis: digital fringe pattern analysis, D. W. Robinson and G. T. Reid, eds. (Institute of Physics, Publishing Ltd., London, 1993), pp. 94-140.
3. I. Yamaguchi and T. Zhang, "Phase-shifting digital holography," Opt. Lett. 22, 1268-1270 (1997).

4. B. Skarman, K. Wozniac, and J. Becker, "Simultaneous 3DPIV and temperature measurement using a New CCD based holographic interferometer," Flow Meas. Instrum. 7, 1-6 (1996).

5. T. M. Kreis, W. P. O. Jüptner, "Principle of digital holography," in Fringe '97: Automatic Processing of Fringe Patterns, W. Jæptner and W. Osten, eds. (Wiley, New York, 1998), pp. 353363.

6. B. Nilsson and T. E. Carlsson, "Direct three-dimensional shape measurement by digital light-in-flight holography," Appl. Opt. 37, 7954-7959 (1998).

7. E. Cuche, F. Bevilacqua, and C. Depeursinge, "Digital holography for quantitative phase contrast imaging," Opt. Lett. 24, 291-293 (1999).

8. D. O. Hogenboom, C. A. Dimarzio, T. J. Gaudette, A. J. Devaney, S. C. Lindberg, "Three-dimensional images generated by quadrature interferometry," Opt. Lett. 23, 783-785 (1998).

9. M. Adams, T. M. Kreis, and W. P. O. Jüptner, "Particle size and position measurement with digital holography," in Optical Inspection and Micromeasurements II, C. Gurecki, ed., Proc. SPIE 3098, 234-240 (1998).

10. E. Cuche, F. Belivacqua, and C. Despeuringe, "Digital holography for quantitative phase-contrast imaging," Opt. Lett. 24, 291-293 (1999).

11. B. Javidi, E. Tajahuerce, "Encrypting three-dimensional information with digital holography," Opt. Lett. 39, 6595-6601 (2000).

12. F. Dubois, L. Joannes, O. Dupont, J. L. Dewandel, and J. C. Legros, "An integrated optical set-up for fluid-physics experiments under microgravity conditions," Meas. Sci. Technol. 10, 934-945 (1999).

13. T. Zhang and I. Yamaguchi, "Three-dimensional microscopy with phase-shifting digital holography," Opt. Lett. 23, 12211223 (1998).

14. F. Dubois, L. Joannes, J.-C. Legros, "Improved threedimensional imaging with digital holography microscope using a partial spatial coherent source," Appl. Opt. 38, 7085-7094 (1999).

15. Y. Takaki and H. Ohzu, "Hybrid holographic microscopy: Visualization of three-dimensional object information by use of viewing angles," Appl. Opt. 39, 5302-5308 (2000).

16. B. Javidi and E. Tajahuerce, "Three-dimensional object recognition by use of digital holography," Opt. Lett. 25, 610-612 (2000).

17. E. Tajahuerce, O. Matoba, and B. Javidi, "Shift-invariant three-dimensional object recognition by means of digital holography," Appl. Opt. 40, 3877-3886 (2001).

18. Y. Frauel, E. Tajahuerce, M.-A. Castro, and B. Javidi, "Distortion-tolerant three-dimensional object recognition with digital holography," Appl. Opt. 40, 3887-3893 (2001).

19. A. V. Oppenheim and J. S. Lim, "The importance of phase in signals,” Proc. IEEE 69, 529-541 (1981).

20. P. Chavel, "Optical noise and temporal coherence," J. Opt. Soc. Am. 70, 935-943 (1980).

21. A. B. Vander Lugt, "Signal detection by complex spatial filtering," IEEE Trans. Inf. Theory IT-10, 139-145 (1964).

22. D. P. Casasent, "Unified synthetic discriminant function computational formulation," Appl. Opt. 23, 1620-1627 (1984).

23. B. V. K. Vijaya Kumar, "Minimum variance synthetic discriminant functions," J. Opt. Soc. Am. 3, 1579-1584 (1986).

24. A. Mahalanobis, B. V. K. V. Kumar, and D. Casasent, "Minimum average correlation energy filters," Appl. Opt. 26, 36333640 (1987).

25. A. Mahalanobis and D. Casasent, "Performance evaluation of minimum average correlation filters," Appl. Opt. 30, 561-572 (1991). 
26. Ph. Réfrégier, "Filter design for optical pattern: multicriteria optimization approach," Opt. Lett. 15, 854-856 (1990).

27. Ph. Réfrégier, "Optimal trade-off filters for noise robustness, sharpness of the correlation peak, and Horner efficiency," Opt. Lett. 16, 829-831 (1991).

28. D. L. Flannery, "Optimal trade-off distortion-tolerant constrained-modulation correlation filters," J. Opt. Soc. Am. A 12, 66-72 (1995).

29. B. Javidi, "Nonlinear joint power spectrum based optical correlation," Appl. Opt. 28, 2358-2367 (1989).

30. A. Vander Lugt and F. B. Rotz, "The use of film nonlinearities in optical spatial filtering," Appl. Opt. 1, 215-222 (1970).

31. Ph. Réfrégier, V. Laude, and B. Javidi, "Nonlinear joint transform correlation: an optimal solution for adaptive image discrimination and input noise robustness," Opt. Lett. 19, 405407 (1994).

32. B. Javidi and D. Painchaud, "Distortion-invariant pattern recognition with Fourier-plane nonlinear filters,” Appl. Opt. 35, 318-331 (1996).

33. M. Guillaume, Ph. Réfrégier, J. Campos, and V. Lashin, "Detection theory approach to multichannel location," Opt. Lett. 22, 1887-1889 (1997).
34. F. Guérault and Ph. Réfrégier, "Unified statistically independent region processor for deterministic and fluctuating targets in nonoverlapping background," Opt. Lett. 23, 412-414 (1998).

35. B. Javidi, F. Parchekani, and G. Zhang, "Minimum-meansquare-error filters for detecting a noisy target in background noise," Appl. Opt. 35, 6964-6975 (1996).

36. F. Dubois, "Automatic spatial frequency selection algorithm for pattern recognition by correlation," Appl. Opt. 32, 43654371 (1993).

37. F. Dubois, "Nonlinear cascaded correlation processes to improve the performances of the automatic spatial-frequencyselective filters in pattern recognition," Appl. Opt. 35, 45894597 (1996).

38. C. Minetti, F. Dubois, "Reduction in correlation filter sensitivity to background clutter using the automatic spatial frequency selection algorithm," Appl. Opt. 35, 1900-1903 (1996).

39. M. Nazarathy and J. Shamir, "Fourier optics described by operator algebra," J. Opt. Soc. Am. 70, 150-159 (1980).

40. S. P. Almeida and J. Kim-Tzong Eu, "Water pollution monitoring using matched spatial filters," Appl. Opt. 15, 510-515 (1976). 